\title{
Impacto da avaliação de risco para úlcera por pressão na adesão da equipe a medidas preventivas recomendadas
}

\author{
Impact of pressure ulcer assessment risk in the team's adherence to \\ recommended preventive measures
}

\author{
Impacto de la evaluación de riesgos de úlceras de presión en la adherencia del \\ equipo en las medidas preventivas recomendadas
}

\author{
Lourdes Aparecida de Castro', Gisela Maria Assis²
}

\begin{abstract}
RESUMO
Introdução: As escalas de predição de risco são amplamente recomendadas para que os protocolos de prevenção de úlceras por pressão sejam aplicados de acordo com o grau de risco do paciente. Objetivo: Avaliar se a implementação da avaliação de risco por meio da escala de Braden influencia na adesão às medidas de prevenção pela equipe de enfermagem. Método: Foi aplicado um checklist com as principais medidas de reposicionamento e conforto recomendadas, antes e depois da implementação da avaliação de risco em um centro de terapia intensiva. Resultados: A adesão foi significativa para elevação de calcâneos, uso de travesseiros, orelhas livres de pressão e fixação de cateteres. A elevação de cabeceira menor que $45^{\circ}$ e o uso de colchões especiais apresentaram piora estatisticamente significante. De maneira geral, os dados isolados por turno influenciaram no resultado geral, com algumas exceções, por exemplo o reposicionamento dos pacientes em até 3 horas não apresentou alteração na avaliação geral antes e após a implantação da avaliação de risco, no entanto, uma das equipes do noturno apresentou uma queda importante no percentual de adesão. Conclusão: Conclui-se que a avaliação de risco por meio da Escala de Braden influencia na adesão às medidas de prevenção pela equipe de enfermagem, porém, observou-se também que outros fatores interferem nesses resultados, tanto positiva como negativamente.
\end{abstract}

DESCRITORES: Úlcera por pressão. Fatores de risco. Educação em saúde. Estomaterapia.

\begin{abstract}
Introduction: Risk prediction scales are widely recommended for the pressure ulcers prevention protocols to be applied according to the patient's degree of risk. Objective: To assess whether the implementation of risk assessment through the Braden scale influences on adherence to preventive measures by the nursing staff. Method: A checklist with key repositioning and comfort recommended measures was applied before and after the risk assessment implementation in an intensive care unit. Results: The adherence was significant for lifting heels, use of pillows, unpressurized ears and fixing catheters. The bedside elevation lower than $45^{\circ}$ and the use of special mattresses worsened statistically significant. In general, the isolated data per turn influenced the overall result, with some exceptions, such as: the repositioning of patients within 3 hours showed no change in the overall assessment before and after the implementation of risk assessment, however, one of the nocturne teams showed a significant drop in the percentage of adherence. Conclusion: The conclusion is that the assessment of risk through the Braden scale influences the nursing staff adherence to prevention actions, but it was observed that other factors affect these results, both positively and negatively.
\end{abstract}

DESCRIPTORS: Pressure ulcer. Risk factors. Health education. Stomatherapy.

${ }^{1}$ Residente Multiprofissional em Atenção Hospitalar do Programa em Urgência e Emergência do Complexo Hospitalar de Clínicas Universidade Federal do Paraná (UFPR) - Curitiba (PR), Brasil.

²Enfermeira Estomaterapeuta TISOBEST; Coordenadora da Comissão de Cuidados com a Pele do Complexo Hospitalar de Clínicas da UFPR - Curitiba (PR), Brasil. Endereço para correspondência: Rua General Carneiro, 181, Alto da Glória - CEP: 80060-900 - Curitiba (PR), Brasil. E-mail: giassis83@gmail.com Artigo recebido em: 01/03/2015 - Aceito para publicação em: 28/03/2016 


\section{RESUMEN}

Introducción: Las escalas para la predicción de riesgo son ampliamente recomendadas para que los protocolos de la prevención de úlceras por presión sean aplicados de acuerdo con el grado de riesgo del paciente. Objetivo: Evaluar si la aplicación de la evaluación de riesgos por medio de la escala de Braden tiene una influencia en la adhesión a las medidas preventivas por parte del equipo de Enfermería. Método: Una lista de verificación fue aplicada a las principales medidas de recolocación y del conforto recomendadas antes y después de la implementación de la evaluación de riesgos en una unidad de cuidados intensivos. Resultados: El cumplimiento fue significativo para la elevación de los talones, uso de las almohadas, orejas sin presión y catéteres de fijación. La elevación de cabecera menor que $45^{\circ}$ y el uso de colchones especiales tuvieron un empeoramiento estadísticamente significativo. En general, los datos aislados influyeron en el resultado global, con algunas excepciones, como el reposicionamiento de los pacientes dentro de tres horas que no mostró cambio en la evaluación general antes y después de la aplicación de la evaluación de riesgos, sin embargo, uno de los equipos de período nocturno presentó una caída significativa en el porcentaje de adherencia. Conclusión: La evaluación del riesgo a través de la escala de Braden influye en el número de miembros del personal de enfermería para la prevención, pero se observaron también otros factores que afectan a estos resultados, tanto positiva como negativamente.

DESCRIPTORES: Úlcera por presión. Factores de riesgo. Educación en salud. Estomaterapia.

\section{INTRODUÇÃO}

A úlcera por pressão (UP) é uma lesão localizada da pele e/ou tecido subjacente, normalmente sobre uma proeminência óssea, em resultado da pressão ou de uma combinação entre esta e forças de cisalhamento. A sua classificação é composta por categorias que variam de I a IV, de acordo com as camadas de tecidos acometidas, além de UP inclassificáveis e suspeita de lesão tissular profunda ${ }^{1}$.

A suscetibilidade individual para o desenvolvimento de UP depende da atuação de fatores extrínsecos (pressão, fricção, cisalhamento e umidade) que se conjugam com as alterações da perfusão tecidual resultantes de fatores intrínsecos (imobilidade, alterações da sensibilidade, incontinência urinária/fecal, alteração do estado de consciência, má perfusão/oxigenação tecidual, estado nutricional) ${ }^{2}$.

Os fatores de risco intrínsecos são inerentes ao indivíduo, ou seja, relacionam-se ao estado físico do paciente. Os principais integrantes desse grupo são imobilidade, presença de incontinência urinária e/ou fecal, alterações na perfusão sanguínea da pele e presença de doenças neurológicas².

O desenvolvimento de UP dificulta o processo funcional de recuperação, além de causar dor e resultar muitas vezes em graves infecções, podendo em casos mais graves ser a causa de quadros de sepse e aumento das taxas de mortalidade ${ }^{3,4}$. Resulta também em custo elevado à instituição e aumento de carga de trabalho diária da equipe de enfermagem ${ }^{5,6}$.

A incidência de UP é um importante indicador de qualidade assistencial na enfermagem, permitindo, assim, a análise dos casos referentes aos pacientes mais suscetíveis, da sua distribuição e dos locais mais frequentes. Tal indicador auxilia na orientação de medidas preventivas, orienta o planejamento e as ações educativas ${ }^{7}$.

No Brasil, estudo realizado em hospital geral universitário evidenciou 39,81\% de incidência de UP. Estudos em unidade de terapia intensiva estimaram incidências de UP de 10,6 a $62,5 \%{ }^{8}$.

Pesquisas demonstram a importância de reduzir a incidência de UP atuando na identificação dos fatores de risco, aplicação de medidas preventivas e educação permanente da equipe multiprofissional ${ }^{9}$.

A fim de garantir a segurança dos pacientes em instituições de saúde, o Ministério da Saúde instituiu o Programa Nacional de Segurança do Paciente (PNSP), sob Portaria no 529, de

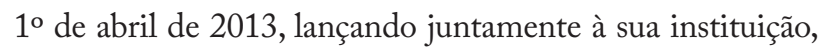
protocolos de prevenção de incidentes, dentre eles as UP ${ }^{10}$. Dentre as medidas citadas no protocolo estão: avaliação dos pacientes em risco, manejo do estado nutricional, inspeção e avaliação diária da pele, manejo da umidade e redistribuição da pressão ${ }^{3}$.

No Brasil, a escala de Braden foi traduzida e validada para a língua portuguesa, sendo a mais bem definida operacionalmente, com alto valor preditivo para o desenvolvimento de UP, permitindo uma avaliação dos vários fatores relacionados à sua ocorrência. Sua aplicação exige do avaliador um exame detalhado das condições do estado do paciente, utilizado para avaliação de risco de UP em pacientes adultos ${ }^{11}$.

Lobosco et al. ${ }^{12}$ ressaltam que é função da enfermagem estabelecer metas, utilizar escalas preditivas de avaliação de 
risco e implantar medidas de prevenção e tratamento das UP através da utilização de seus conhecimentos específicos, estabelecendo um processo avaliativo contínuo, preservando então a integridade da pele do paciente.

Diante do conhecimento a respeito da efetividade das medidas de prevenção para UP e da capacidade preditiva da escala de Braden para identificar pacientes em risco e direcionar as medidas, buscou-se avaliar o impacto causado pela avaliação diária de risco de pacientes críticos, na adesão da equipe de enfermagem para aplicação de medidas de prevenção a pacientes em risco.

\section{MÉTODOS}

Tratou-se de uma pesquisa exploratória descritiva com abordagem quantitativa. Realizada em um centro de terapia intensiva (CTI) de um hospital universitário da cidade de Curitiba, Paraná, sendo que os dados foram coletados no período de 16 a 18 de novembro do ano de 2015. A pesquisa foi aprovada pelo Comitê de Ética em Pesquisa com Seres Humanos da instituição (Parecer no 45141415.0.0000.0096) e todos os preceitos da Resolução 466/2012 do Conselho Nacional de Saúde foram devidamente seguidos.

A instituição onde o estudo foi realizado conta com uma Comissão de Cuidados com a Pele (CCP) que tem, entre suas atividades, auditorias periódicas para avaliação da aplicação das medidas de prevenção. Esses dados foram coletados por meio de checklists contendo as principais medidas de prevenção recomendadas pelo European Pressure Ulcer Advisory Panel e National Pressure Ulcer Advisory Panel (EPUAP/NPUAP), que são preenchidos informando se cada medida está ou não aplicada adequadamente em cada um dos pacientes com mobilidade reduzida.

Para obtenção dos dados de aplicação das medidas anteriores à implantação da avaliação diária de risco foram utilizados dados tabulados pela CCP. De posse dos dados a autora do estudo realizou uma capacitação com todos os enfermeiros do CTI, sanando dúvidas em relação à aplicação da escala de Braden para avaliação de risco. Ficou acordado que a partir da capacitação, além dos pacientes serem avaliados diariamente por um enfermeiro quanto ao risco para UP, pela escala de Braden, os pacientes de alto risco teriam a porta de seus boxes sinalizados, a fim de alertar a equipe para a aplicação das medidas de prevenção.
Depois de transcorridos 30 dias com a nova rotina estabelecida, a autora do estudo realizou a auditoria de aplicação das medidas preventivas. Essa auditoria foi realizada em todos os boxes do CTI, e repetidos em todos os turnos, sendo manhã, tarde e três noites, por possuírem equipes distintas.

As medidas preventivas avaliadas na auditoria foram: reposicionamento do paciente em até 3 horas, lateralização menor que $90^{\circ}$, elevação de cabeceira com angulação menor do que $45^{\circ}$, calcâneos livres com uso de apoio sob as panturrilhas, uso de travesseiro para apoio da cabeça, orelhas livres de pressão, uso de colchão especial e fixação correta de cateteres. Para avaliação do reposicionamento, a pesquisadora voltava nos boxes três horas depois da primeira avaliação para ver se a posição inicial havia sido alterada.

Os dados coletados foram tabulados em planilha Excel e analisados estatisticamente pelo programa IBM SPSS Statistics v.20.0. Foram comparados os dados de percentual de adesão já existentes com os novos dados coletados após implementação sistematizada da avaliação de risco. Os resultados obtidos para cada variável foram descritos por frequências e percentuais. Para as comparações entre as avaliações pré e pós-implementação da avaliação de risco e comparações entre turnos foi considerado o teste do $\chi_{2}$ ou $o$ teste exato de Fisher, sendo que valores de $\mathrm{p}<0,05$ indicaram significância estatística.

\section{RESULTADOS}

O número de avaliações realizadas foi de 68. Conforme demonstrado na Tabela 1 , não houve diferença estatisticamente significativa para reposicionamento dos pacientes anterior e posteriormente à implantação da avaliação de risco. A lateralização dos pacientes em um ângulo menor do que $90^{\circ}$ se manteve inalterada, porém, vale ressaltar que apenas em 17 das 68 avaliações os pacientes se encontravam em decúbito lateral. O que dificultou a aplicação de testes estatísticos.

As medidas preventivas que apresentaram sua adesão aumentada, com significância estatística, após a implantação da avaliação de risco foram: elevação dos calcâneos com apoio sob as panturrilhas, uso de travesseiros na cabeça ao invés de lençóis ou cobertores dobrados, orelhas livres de pressão do cadarço de fixação do tubo orotraqueal e fixação de cateteres vesicais e nasogástricos ou enterais. 
A elevação de cabeceira com uma elevação menor do que $45^{\circ}$ e o uso de colchões especiais (pneumáticos ou colchão piramidal) foram itens que apresentaram piora da primeira para a segunda avaliação, com significância estatística.

A Tabela 2 demonstra que equipes de alguns turnos apresentaram resultados estatisticamente significativos em determinadas medidas de prevenção isoladamente. De maneira geral, os dados isolados por turno influenciaram nos resultados gerais. Porém, vale observar que alguns turnos destoaram dos resultados gerais, por exemplo, o reposicionamento dos pacientes em até 3 horas não apresentou alteração na avaliação geral antes e após a implantação da avaliação de risco, no entanto, uma das equipes do noturno apresentou uma queda significativa no percentual de adesão.

Apesar do combinado com as equipes de que os pacientes que pontuassem como alto risco para UP fossem sinalizados na porta do box como risco para UP, observou-se que apenas
11 leitos avaliados estavam sinalizados, embora diversos dos não sinalizados tivessem pontuado como alto risco.

Avaliou-se se a sinalização do box influenciou na aplicação das medidas de prevenção, ou seja, se os pacientes sinalizados como estando em risco receberam mais medidas preventivas do que os não sinalizados. A Tabela 3 demonstra que apenas para o item elevação de calcâneo a sinalização do box apresentou influência na adesão.

\section{DISCUSSÃO}

A assistência direta embasada na prevenção e no tratamento de UP tem a enfermagem como grande responsável ${ }^{4}$. $\mathrm{O}$ aumento no percentual de adesão da equipe às medidas preventivas demonstrou ser significativo em alguns dos itens avaliados, conforme apresentado anteriormente nos resultados.

Tabela 1. Percentual de adesão às medidas de prevenção para úlceras por pressão antes e depois da implantação da avaliação de risco pela escala de Braden. Curitiba, PR, 2015.

\begin{tabular}{|c|c|c|c|}
\hline Medidas de prevenção & $\begin{array}{l}\text { Pré-implantação da } \\
\text { avaliação de risco (\%) }\end{array}$ & $\begin{array}{l}\text { Pós-implantação da } \\
\text { avaliação de risco (\%) }\end{array}$ & Valor $p$ \\
\hline Reposicionamento & 53,8 & 42,6 & 0,226 \\
\hline Lateralização $<90^{\circ}$ & 100,0 & 100,0 & 1,000 \\
\hline Elevação de cabeceira <45 & 100,0 & 84,6 & 0,002 \\
\hline Calcâneos livres & 27,7 & 48,5 & 0,020 \\
\hline Uso de travesseiro & 32,3 & 73,5 & $<0,001$ \\
\hline Orelhas livres de pressão & 56,9 & 91,0 & $<0,001$ \\
\hline Uso de colchão especial & 35,4 & 1,5 & $<0,001$ \\
\hline Cateteres bem fixados & 26,2 & 60,6 & $<0,001$ \\
\hline
\end{tabular}

Para as comparações entre as avaliações pré e pós-implementação da avaliação de risco foi considerado o teste do $\chi^{2}$ ou o teste exato de Fisher, sendo que valores de $p<0,05$ indicaram significância estatística.

Tabela 2. Percentual de adesão às medidas de prevenção paraúlceras por pressão antes e depois da implantação da avaliação de risco, pela escala de Braden, categorizadas por turno de trabalho. Curitiba, PR, 2015.

\begin{tabular}{|c|c|c|c|c|c|c|c|c|c|c|}
\hline \multirow{2}{*}{ Item avaliado } & \multicolumn{2}{|c|}{ Manhã } & \multicolumn{2}{|c|}{ Tarde } & \multicolumn{2}{|c|}{ Noite 1} & \multicolumn{2}{|c|}{ Noite 2} & \multicolumn{2}{|c|}{ Noite 3} \\
\hline & Pré & Pós & Pré & Pós & Pré & Pós & Pré & Pós & Pré & Pós \\
\hline Reposicionamento & 53,9 & 46,2 & 42,9 & 50,0 & 71,4 & 50,0 & $72,7^{*}$ & $23,1 *$ & 30,8 & 42,9 \\
\hline $\begin{array}{l}\text { Elevação de } \\
\text { cabeceira <450 }\end{array}$ & 100,0 & 92,3 & 100,0 & 100,0 & 100,0 & 92,9 & 100,0 & 69,2 & 100,0 & 71,4 \\
\hline Calcâneos livres & 38,5 & 38,5 & $14,3^{*}$ & $64,3^{*}$ & 28,6 & 28,6 & 18,2 & 53,9 & 38,5 & 57,1 \\
\hline Uso de travesseiro & $38,5 *$ & $84,6 *$ & 50,0 & 71,4 & $21,4^{*}$ & $71,4^{*}$ & 27,3 & 69,2 & $23,1 *$ & $71,4^{*}$ \\
\hline $\begin{array}{l}\text { Orelhas livres de } \\
\text { pressão }\end{array}$ & 46,2 & 84,6 & $42,9 *$ & $92,3 *$ & 71,4 & 85,7 & $63,6 *$ & $100 *$ & 61,5 & 92,9 \\
\hline $\begin{array}{l}\text { Uso de colchão } \\
\text { especial }\end{array}$ & $38,5 *$ & $0,0 *$ & 35,7 & 7,1 & $35,7 *$ & 0,0 * & $36,4^{*}$ & $0,0^{*}$ & $30,8^{*}$ & $0,0 *$ \\
\hline $\begin{array}{l}\text { Cateteres bem } \\
\text { fixados }\end{array}$ & 30,8 & 61,5 & 21,4 & 58,3 & 28,6 & 57,1 & 27,3 & 69,2 & 23,1 & 57,1 \\
\hline
\end{tabular}

Os valores estão expressos em \%; *valores com significância estatística na diferença nos dados pré e pós-implantação da escala de Braden. Para as comparações entre turnos foi considerado o teste do $\chi^{2}$ ou o teste exato de Fisher, sendo que valores de p<0,05 indicaram significância estatística. 
Lobosco et al. ${ }^{12}$ ressaltam que é função da enfermagem estabelecer metas, utilizar escalas preditivas de avaliação de risco e implantar medidas de prevenção e tratamento das UP através da utilização de seus conhecimentos específicos, estabelecendo um processo avaliativo contínuo, preservando então a integridade da pele do paciente.

Quanto à adesão das equipes no item reposicionamento, observou-se redução da adesão quando comparado ao período anterior à implantação da avaliação de risco, embora essa redução não tenha atingido significância estatística. Pensa-se que essa redução pode ser atribuída ao fato do período pós-implementação da avaliação de risco coincidir com uma fase institucional de entrada de novos profissionais de nível médio e certa desmotivação da equipe pela necessidade de transferência pela substituição de auxiliares por técnicos de enfermagem nesse serviço.

O EPUAP/NPUAP ${ }^{1}$ enfatiza que todos os esforços devem ser feitos para redistribuir a pressão sobre a pele, seja pelo reposicionamento a cada duas horas ou pela utilização de superfícies de redistribuição de pressão. Ressalta ainda que esse tempo estimado em uma única posição é o máximo de tempo recomendado para pacientes com capacidade circulatória normal. O tempo de intervalo utilizado neste estudo para o reposicionamento dos pacientes foi de três horas, dando com isso uma hora de tolerância em caso de intercorrência que impedisse o reposicionamento em duas horas.

Segundo o Protocolo do Ministério da Saúde ${ }^{3}$ o principal objetivo na prevenção de UP está associado à redistribuição da pressão, especialmente sobre as proeminências ósseas. O reposicionamento dos pacientes em risco para desenvolvimento de UP é fator determinante para aliviar a pressão sobre áreas suscetíveis, reduzindo assim o surgimento das lesões.
Quanto à angulação de lateralização e elevação de cabeceira, o guideline utilizado como referência apresenta que o ideal é uma inclinação de $30^{\circ}$ para posições laterais (alternadamente lado direito, dorsal e lado esquerdo), bem como elevação de cabeceira de até $30^{\circ 1}$. Quando analisado globalmente, observa-se uma adesão de $100 \%$ na lateralização com angulação menor do que $90^{\circ}$, porém, nenhuma significância estatística, uma vez que apenas 17 pacientes estavam em decúbito lateral durante as avaliações, reforçando a falha de reposicionamento.

Diante de um percentual de piora significativa no uso de colchões e elevação de cabeceira menor do que $45^{\circ}$ graus, supõe-se novamente a atuação de outros fatores nos resultados finais. Ressalta-se novamente a situação institucional do momento e cita-se que durante a implantação da avaliação de risco, diversos colchões pneumáticos antes disponíveis deixaram de funcionar devido a furos e falha técnica, e que a engenharia clínica e a manutenção do hospital não estavam aceitando os mesmos para conserto. Sendo assim, pode-se dizer que a disponibilidade de recursos materiais influenciou diretamente nos resultados.

O EPUAP/NPUAP recomenda elevar a cabeceira da cama no máximo em $30^{\circ}$, limitando o tempo de cabeceira elevada, pois o corpo do paciente pode escorregar no leito, ocasionando fricção, cisalhamento e aumento da pressão no sacro e no cóccix 5 .

O protocolo do Ministério da Saúde ${ }^{3}$ diz que, apesar de ocorrer a redução de cisalhamento no posicionamento da cabeceira até $30^{\circ}$, para os pacientes em ventilação mecânica e traqueostomizados com ventilação não invasiva, é recomendado decúbito acima de $30^{\circ}$ para a prevenção de pneumonia associada à ventilação (PAV). Diante do exposto, considerando as características dos pacientes internados nos locais de

Tabela 3. Percentual de adesão a medidas de prevenção de úlceras por pressão pela equipe de enfermagem, anteriormente e após a implantação diária de avaliação de risco pela escala de Braden, sem sinalização de risco e com sinalização de risco. Curitiba, PR, 2015.

\begin{tabular}{|c|c|c|c|}
\hline Item avaliado & $\begin{array}{l}\text { Sem sinalização } \\
\text { de risco (\%) }\end{array}$ & $\begin{array}{l}\text { Com sinalização } \\
\text { de risco (\%) }\end{array}$ & Valor $p$ \\
\hline Reposicionamento & 47,4 & 18,2 & 0,100 \\
\hline Elevação de cabeceira <450 & 83,3 & 90,9 & 1,000 \\
\hline Calcâneos livres & 42,1 & 81,8 & 0,021 \\
\hline Uso de travesseiro & 70,2 & 90,9 & 0,265 \\
\hline Orelhas livres de pressão & 91,1 & 90,9 & 1,000 \\
\hline Uso de colchão especial & 1,8 & 0,0 & 1,000 \\
\hline Cateteres bem fixados & 57,1 & 80,0 & 0,293 \\
\hline
\end{tabular}

Para as comparações entre as avaliações foi considerado o teste do $\chi^{2}$ ou exato de Fisher, sendo que valores de $p<0,05$ indicaram significância estatística. 
estudo, optou-se por orientar a elevação de cabeceira menor do que $45^{\circ}$. Lembrando que o envolvimento da equipe de enfermagem na prevenção da PAV pode ter influenciado na não adesão, além de haver uma equipe multiprofissional não completamente capacitada na prevenção de UP na rotina diária do tratamento desses pacientes, sendo que seus aspectos clínicos são avaliados separadamente.

Tratando-se ainda de mobilidade do paciente, se essa se encontra comprometida e a pressão não está redistribuída, a circulação pode ser diretamente afetada, levando ao surgimento de UP. Por isso é recomendada a utilização de superfícies de apoio específicas (como colchões e travesseiros), pois redistribuem a pressão que o corpo exerce sobre a pele e os tecidos subcutâneos ${ }^{3}$.

O EPUAP/NPUAP ${ }^{1}$ recomenda o uso de uma superfície de apoio dinâmica (colchão ou sobreposição) em indivíduos com alto risco de desenvolver UP, principalmente quando não é possível o reposicionamento manual pela equipe de enfermagem, pois isso pode auxiliar na distribuição de carga.

Em relação à prevenção de UP nos calcâneos, de acordo com o Institute for Healthcare Improvement ${ }^{13}$, os materiais utilizados na prevenção de UP devem ser elevados de tal forma que o peso da perna seja distribuído ao longo da sua parte posterior, sem colocar pressão sobre o tendão de Aquiles. E é importante utilizar a almofada ou travesseiro sob panturrilhas, a fim de elevar completamente os calcâneos e mantê-los suspensos. Os resultados apresentaram significância estatística positiva, exceto no período da manhã e noite 1 , onde mantiveram-se inalterados. Esse item apresentou relação direta com a sinalização de risco fixada nos leitos dos pacientes.

O Ministério da Saúde dispõe que é de grande importância o uso de travesseiros, pois quando utilizados de forma apropriada podem expandir a superfície que suporta o peso e, além disso, podem ser utilizados para auxiliar a redistribuição da pressão.

É citado pelo Ministério da Saúde ${ }^{3}$ que travesseiros são de fácil acesso, e nas unidades onde foi realizado o estudo foram encontrados disponíveis para todos os leitos do CTI durante o período da realização da pesquisa, o que pode ter influenciado positivamente nos resultados encontrados, demonstrando novamente a influência de recursos materiais.

No item orelhas livres de pressão, foram avaliados todos os dispositivos que viessem a causar algum tipo de pressão, como por exemplo o cateter de oxigênio, cadarço de fixação para névoas e tubo endotraqueal. Vale ressaltar que o fato de existirem formas alternativas disponíveis no serviço (utilização de eletrodos sob o cadarço) para fixação dos dispositivos citados, o percentual de adesão a tal medida preventiva apresentou aumento expressivo, o que impactou tanto nos resultados globais quanto nos analisados separadamente.

De acordo com o EPUAP/NPUAP ${ }^{1}$, diversos tipos de dispositivos têm sido identificados como causadores de UP (por exemplo, cateteres, tubos de oxigênio, tubulação do ventilador, etc.). Enfatiza-se, então, a importância de monitorizar a pele do paciente quanto a possíveis danos causados por pressão, por esses dispositivos. No estudo foi verificada significância na adesão da medida preventiva de fixação de drenos e cateteres, visto que durante a pesquisa foi levado em consideração se todos os dispositivos do paciente estavam fixados corretamente (acesso venoso periférico, cateter venoso central, cateter de pressão arterial média, drenos, cateter vesical de demora, etc.).

\section{CONCLUSÃO}

Pode-se concluir que a implantação da avaliação de risco pela escala de Braden influenciou na adesão da equipe a alguns itens de medidas preventivas para UP, especialmente quando a sinalização dos boxes foi seguida pelos enfermeiros. A sinalização pode atuar como um lembrete para aplicação de medidas já conhecidas.

Fatores como disponibilidade de recursos financeiros e desmotivação da equipe por questões institucionais interferiram nos resultados, dificultando a avaliação do impacto isolado de uma sistematização de avaliação de risco.

Ainda quanto a limitações do estudo, pode ser citado o fato dos enfermeiros não terem cumprido o combinado de realizar a sinalização nos leitos para os pacientes com alto risco de desenvolvimento de UP. Ressalta-se a necessidade de participação ativa dos profissionais enfermeiros nas atividades de capacitação, pois o turno de trabalho cujo enfermeiro responsável da unidade apresentou um maior envolvimento nas discussões durante as atividades de capacitação foi o que apresentou resultados mais expressivos de melhora na adesão às medidas.

Ressalta-se que a avaliação de risco, apesar de se mostrar efetiva, não pode ser a única estratégia para envolver a equipe na prevenção de UP e na segurança do paciente; todos os itens trabalhados neste estudo precisam ser exaustivamente discutidos por meio de educação permanente da equipe. Faz-se necessária também a realização de trabalhos futuros e maior número de avaliações por turno de trabalho, depois de implantada a rotina de sinalização no leito dos pacientes que apresentarem um maior risco de desenvolvimento de UP. 


\section{REFERÊNCIAS}

1. National Pressure Ulcer Advisory Panel, European Pressure Ulcer Advisory Panel and Pan Pacific Pressure Injury Alliance. Prevention and treatment of pressure ulcers: quick reference guide. Washington DC: National Pressure Ulcer Advisory Panel; 2014.

2. Pinto JR. Proposta de protocolo clínico para tratamento de feridas crônicas. Trabalho de Conclusão de Curso (Especialização em Atenção Básica em Saúde da Família). Teófilo Otoni: Universidade Federal de Minas Gerais.

3. Ministério da Saúde, Anvisa, Fiocruz. Anexo 02: Protocolo para prevenção de úlcera por pressão. Brasília: Ministério Da Saúde; 2013.

4. Jaul E. Assessment and management of pressure ulcers in the elderly: current strategies. Drugs Aging. 2010;27(4):311-25.

5. American Medical Directors Association. Pressure ulcers in the long-term care setting. Columbia: AMDA; 2008.

6 Bergquist-Beringer SB, Gajewski B, Dunton N, Klaus S. The reliability of the National Database of Nursing Quality Indicators pressure ulcer indicator: a triangulation approach. J Nurs Care Qual. 2011;26(4):292-301.

7. Moura GMSS, Juchem BC, Falk MLR, Magalhães AMM, Suzuki LM. Construção e implantação de dois indicadores de qualidade assistencial de enfermagem. Rev Gaúcha Enferm. 2009;30(1):136-40

8. Rogenski NMB, Santos VLCG. Estudo sobre a incidência de úlceras por pressão em um hospital universitário. Rev Latino-Am Enfermagem. 2005;13(4):474-80.

9. Bavaresco T, Medeiros RH, Lucena AF. Implantação da Escala de Braden em uma unidade de terapia intensiva de um hospital universitário. Rev Gaúcha Enferm. 2011;32(4):703-10.

10. Brasil. Portaria $n^{\circ} 529$, de $1^{\circ}$ de abril de 2013. Institui o Programa Nacional de Segurança do Paciente (PNSP). Brasília: Ministério da Saúde; 2013.

11. Silva EWNL, Araújo RA, Oliveira EC, Falcão VTFL. Aplicabilidade do protocolo de prevenção de úlcera por pressão em unidade de terapia intensiva. Rev Bras Ter Intensiva. 2010;22(2):175-85

12. Lobosco AAF, Vasconcelos DDS, Almeida LMC, Feliciano, GD. O enfermeiro atuando na prevenção das úlceras de pressão. Enferm Global. 2008;13:1-15.

13. Institute for Healthcare Improvement. How-to guide: prevent pressure ulcers. Cambridge: Institute for Healthcare Improvement; 2011. 